\title{
Uncovering beam position monitor noise at the Relativistic Heavy Ion Collider
}

\author{
X. Shen ${ }^{*}$ and S. Y. Lee \\ Department of Physics, Indiana University, Bloomington, Indiana 47405, USA \\ M. Bai \\ Brookhaven National Laboratory, Upton, New York 11973, USA \\ (Received 16 August 2014; published 12 January 2015)
}

\begin{abstract}
We apply the independent component analysis (ICA) algorithm to uncover intrinsic noise in the beam position monitor (BPM) system. Numerical simulations found that ICA is efficient in the BPM noise estimation. The ICA algorithm is applied to the turn-by-turn data at the Relativistic Heavy Ion Collider. We found the distribution of the BPM noise level, which is consistent with the Johnson-Nyquist thermal noise model. The ICA analysis of turn-by-turn data can be used in neuronetwork feasibility of monitoring a storage ring parasitically.
\end{abstract}

DOI: 10.1103/PhysRevSTAB.18.014002

PACS numbers: 29.85.Fj, 29.90.+r

\section{INTRODUCTION}

Independent component analysis (ICA) is a powerful blind source separation method [1]. It is highly efficient in separating narrow-band source signals from sampled data. Numerical simulations indicate that the ICA can be used to uncover nonlinear modes for sextupole strength measurement [2], vertical dispersion function measurement, and emittance correction [3]. In actual experimental data analysis, ICA has been successfully utilized to extract characteristic beam motion signals from turn-by-turn (TBT) data for beam optics measurement at the Fermilab booster [4], Los Alamos Proton Storage Ring [5], and Relativistic Heavy Ion Collider (RHIC) [6]. Compared to the singular value decomposition (SVD) -based principle component analysis [7], ICA is more robust in the presence of noise [4]. ICA can also be used to discover intrinsic problems of beam position monitors (BPMs). For example, a bad BPM will appear as a mode in ICA with spatial function pointing to the position of the BPMs [4]. This paper addresses the question of uncovering intrinsic BPM noise from the TBT BPM data.

BPMs are commonly used to record the beam centroid motion. At the RHIC, there are 160 BPMs for each plane in each of the blue and yellow rings: 72 dual-plane BPMs in the insertion regions (IRs) and 176 single-plane modules in the arcs. Each BPM can acquire 1024 or 4096 consecutive TBT beam position data. For a linear BPM system, the acquired TBT data is a linear superposition of narrow-band

\footnotetext{
*Present address: SLAC National Accelerator Laboratory, Menlo Park, California 94025, USA.

xshen@slac.stanford.edu

Published by the American Physical Society under the terms of the Creative Commons Attribution 3.0 License. Further distribution of this work must maintain attribution to the author $(s)$ and the published article's title, journal citation, and DOI.
}

beam motions, each of which has its characteristic frequency, e.g., betatron oscillation, synchrotron oscillation, and nonlinear oscillations. Inevitably, there is broadband noise in the TBT data due to digitization, electronic noise, etc. Sometimes, a few BPMs may even be malfunctioning. SVD has been used to identify faulty BPMs from TBT data [8]. A detailed study of the intrinsic BPM noise is critical for the successful understanding of beam dynamics in high energy accelerators [9].

At RHIC, we applied ICA to TBT BPM data driven by ac dipoles [10] for optics measurement and correction [6]. This paper presents a study of using ICA for RHIC BPM noise estimation. In Sec. II, we carry out detailed simulations to prove that the ICA algorithm can be used to extract the BPM noise. In Sec. III, the algorithm to estimate RHIC BPM noise with experimental TBT BPM data is applied. A model for these noise data is also discussed. The conclusion is given in Sec. IV.

\section{PRINCIPLE OF BPM NOISE ESTIMATION USING ICA}

The TBT BPM data are organized into a data matrix form suitable for ICA analysis:

$$
\mathbf{X}=\left(\begin{array}{cccc}
x_{1}(1) & x_{1}(2) & \cdots & x_{1}(P) \\
x_{2}(1) & x_{2}(2) & \cdots & x_{2}(P) \\
\vdots & \vdots & \ddots & \vdots \\
x_{M}(1) & x_{M}(2) & \cdots & x_{M}(P)
\end{array}\right)
$$

where $x_{i}(j)$ is the reading of the $i$ th BPM at the $j$ th turn and the dc component has been removed from each BPM channel. 

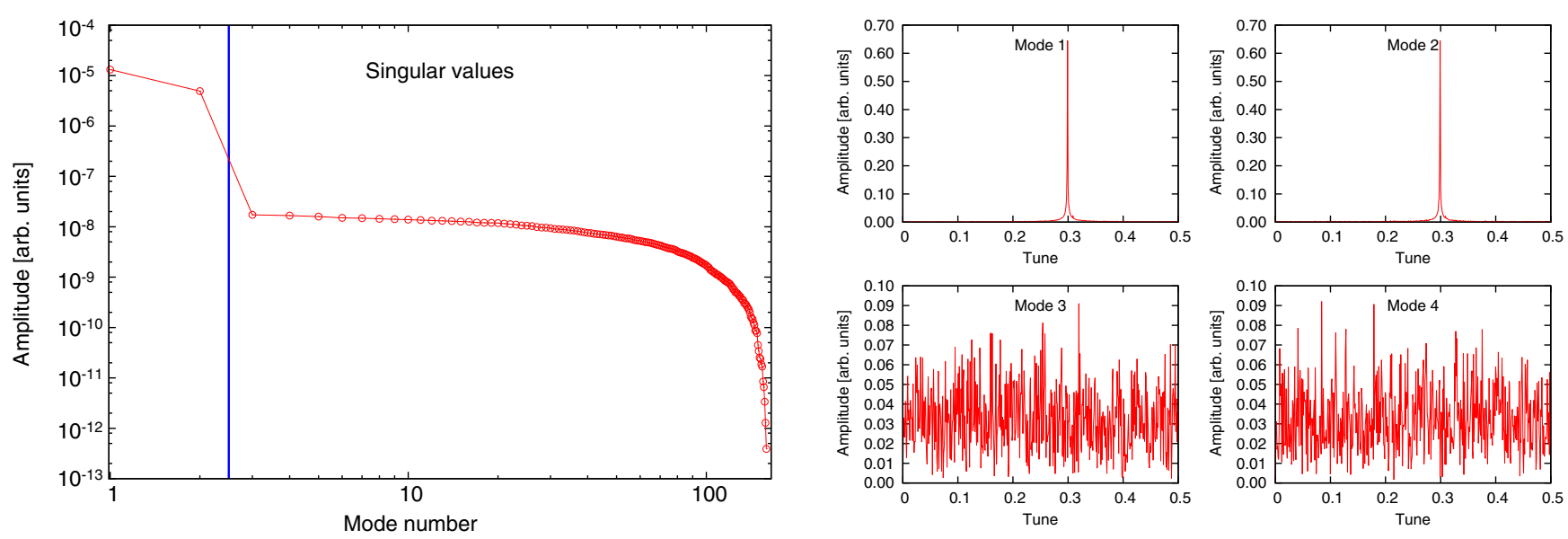

FIG. 1. Left: Typical distribution of singular values for the simulated TBT BPM data. The cutoff mode number is chosen to be $n_{c}=2$. Right: Typical spectrum of temporal functions from mode 1 to 4 for the simulated turn-by-turn data. Modes 1 and 2 correspond to betatron motion, while modes 3 and 4 are noiselike.

When ICA is successfully carried out, the data matrix can be decomposed into modes:

$$
\mathbf{X}=\sum_{i=1}^{n_{c}} \mathbf{A}_{i} \mathbf{s}_{i}+\mathbf{N}
$$

where the column vector $\mathbf{A}_{i}$ is called the $i$ th spatial function, the row vector $\mathbf{s}_{i}$ is the $i$ th temporal function, $n_{c}$ is the cutoff mode number, and $\mathbf{N}$ is the noise data matrix. The combination of $\mathbf{A}_{i}$ and $\mathbf{s}_{i}$ is called the $i$ th mode. The product of $\mathbf{A}_{i}$ and $\mathbf{s}_{i}$ reconstructs a mode of beam motion, such as betatron oscillation, synchrotron oscillation, and nonlinear beam oscillations. A proper choice of cutoff mode number $n_{c}$ provides a better description of beam motions from the data matrix $\mathbf{X}$. At the same time, the extracted noise $\mathbf{N}: \mathbf{N}=\mathbf{X}-\sum_{i=1}^{n_{c}} \mathbf{A}_{i} \mathbf{s}_{i}$ will be free from a coherent signal.

BPM noise is usually assumed to be Gaussian distributed with zero mean, zero unequal-time correlation. The equal time covariance matrix $\mathbf{C}_{\mathbf{N}}$ of noise data $\mathbf{N}$ is $\mathbf{C}_{\mathbf{N}}=\left\langle\mathbf{N} \mathbf{N}^{T}\right\rangle=\operatorname{diag}\left(\sigma_{1}^{2}, \sigma_{2}^{2}, \ldots, \sigma_{M}^{2}\right)$, which gives an estimation of noise at $M$ BPMs.

We carry out numerical simulations to evaluate the performance of the ICA algorithm for BPM noise estimation in the following steps: (i) TBT BPM data were generated by single particle tracking for 1024 turns for a RHIC lattice; (ii) each BPM was assigned a standard deviation, Gaussian white noise was then generated by using the assigned standard deviation added to the TBT BPM data to simulate the raw TBT BPM data, and noise at each BPM was generated by using a unique random seed; (iii) the ICA algorithm described above was used to extract the BPM noise. In this simulation, the standard noise deviation assigned at each BPM was sampled from a uniform distribution in a range between 0 and $120 \mu \mathrm{m}$, which is enough to cover the expected noise level for RHIC BPMs.

The left plot in Fig. 1 shows a typical singular value distribution for the simulated TBT BPM data. The first two singular values correspond to the betatron motion, larger than the other modes by about 3 orders of magnitude. The spectra of the temporal functions of the first four modes are shown in the right plot in Fig. 1, in which the temporal functions of the first two modes exhibit characteristic frequency of the betatron tune, while the other two are noiselike.

From the distribution of the singular values, we choose the cutoff mode number to be $n_{c}=2$. The left plot in Fig. 2 shows assigned and estimated standard deviations for noise at all 160 BPMs. The right plot in Fig. 2 shows the estimated standard deviations versus the assigned values. The error bar shows a small variation of ten different random seeds. This result proves that the ICA algorithm can accurately capture the BPM noise level.

\section{RHIC BPM NOISE ESTIMATION FROM EXPERIMENTAL DATA}

Since the ICA algorithm can provide a good estimation of BPM noise, we apply it to experimental TBT BPM data taken during the RHIC 2013 polarized proton (PP) and $2014 \mathrm{Au}^{79+}$ operation in the yellow ring. Note that the same algorithm can be applied to the blue ring. Since the results are similar, we will report only those of the yellow ring.

The right plot in Fig. 3 shows a typical distribution of singular values in one set of experimental data taken for PP collision with an intensity of $1.2 \times 10^{11}$. The corresponding spectrum of temporal functions of the first four dominating modes are shown in the right plot in Fig. 3. Modes 1 and 2 correspond to betatron motion, while modes 3 and 4 are related to synchrotron motion with low frequencies. BPM 

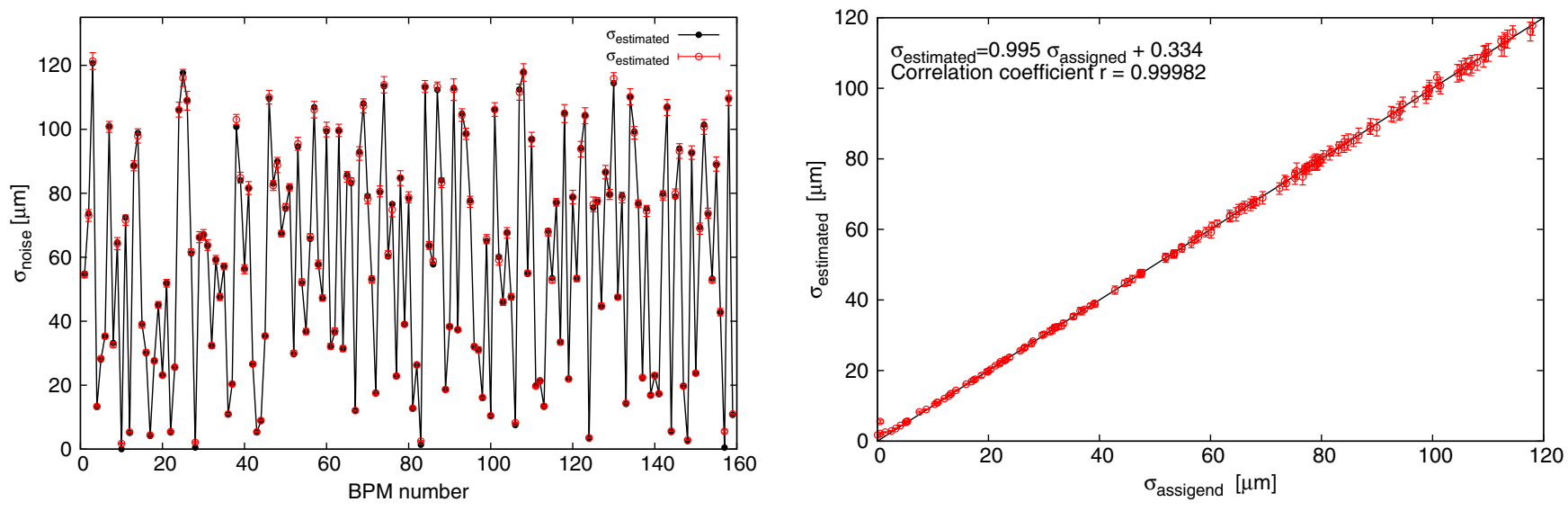

FIG. 2. Left: Assigned and estimated standard deviations of noise at different BPMs. Error bars reflect the standard deviation of ten estimations in which each estimation was against TBT BPM data with noise generated by a unique random seed. Right: Estimated versus assigned standard deviations of BPM noise.
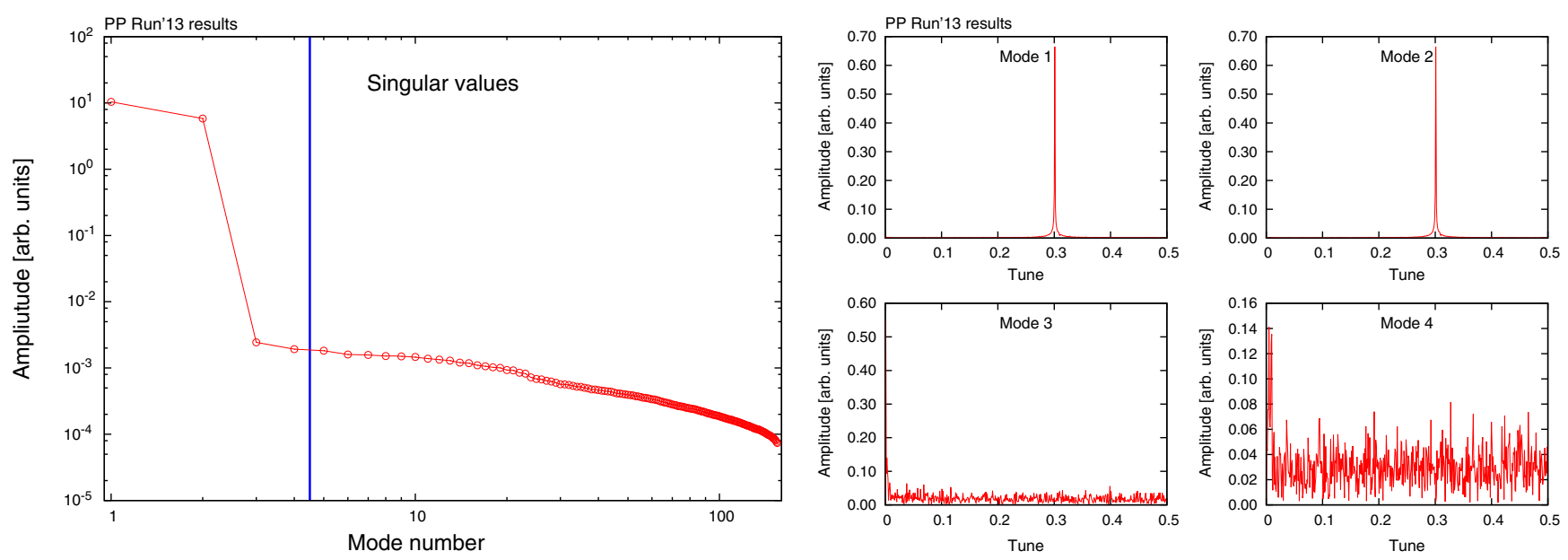

FIG. 3. Typical distribution of singular values for one set of experimental data of PP collision with an intensity of $1.2 \times 10^{11}$. The cutoff mode number is chosen to be $n_{c}=4$. Right: Typical spectrum of temporal function for the corresponding modes $1-4$. Modes 1 and 2 correspond to betatron motion, while modes 3 and 4 are related to the low-frequency synchrotron motions.

noise is then estimated with $n_{c}=4$. Figure 4 shows the estimated noise level at horizontal BPMs (top plot) and vertical BPMs (lower plot) for different operations with various bunch intensities: PP collision at $0.8 \times 10^{11}$ and $1.2 \times 10^{11}$ and Au-Au collision at $0.48 \times 10^{9}, 0.90 \times 10^{9}$, and $1.40 \times 10^{9}$ particles per bunch, respectively. Note that a larger beam current gives rise to a smaller average BPM noise. The largest noise occur at the BPMs next to triplet quadrupoles in the IRs. The distribution of arc BPM noise shows a peak at the center of the arcs and is symmetric about the arc center.

Figure 5 shows histograms of the estimated BPM noise corresponding to the case of $\mathrm{Au}-\mathrm{Au}$ operation with a bunch intensity of $0.48 \times 10^{9}$ in Fig. 4. The four types of distributions in the histograms are consistent with the four types of RHIC BPMs whose mechanical details are listed in
Table I. Histograms for the other estimated noise in Fig. 4 also show similar distributions.

BPM noise may arise from quantization error and thermal noise. Since the RHIC BPM uses a 16-bit digitizing system, its resolution is typically $1 \mu \mathrm{m}$ [11]. The quantization error in the estimated BPM noise is thus negligible. The root mean square thermal noise in the induced voltage of a BPM plate is $\sigma_{v}=\sqrt{4 k_{\mathrm{B}} T R \Delta f}$ [12], where $k_{\mathrm{B}}$ is the Boltzmann constant, $T$ is temperature, $\Delta f$ is sampling bandwidth, and $R$ is BPM impedance. RHIC BPM noise was reduced by a $20 \mathrm{MHz}$ low pass filter [11].

The beam position is usually measured by using the difference-over-sum signal. For example, horizontal beam position $x$ is [13]

$$
x=\frac{d}{4} \frac{V_{R}-V_{L}}{V_{R}+V_{L}},
$$




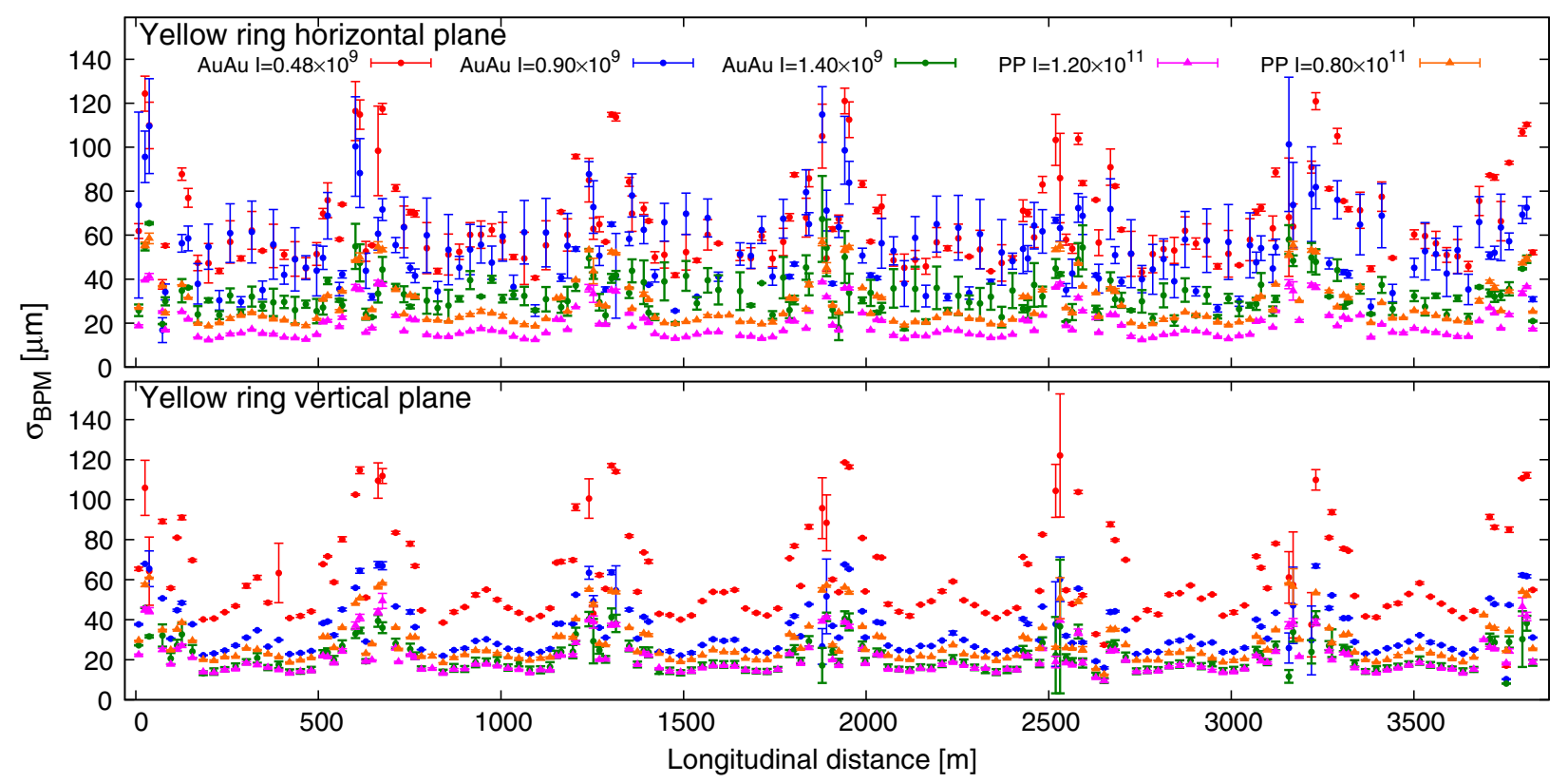

FIG. 4. Estimated standard deviations of BPM noise from TBT BPM data of 2013 polarized proton operation with two different intensities and $2014 \mathrm{Au}^{79+}$ operation with three different intensities. Error bars reflect statistical standard deviations of estimated noise over three sets of experimental data.
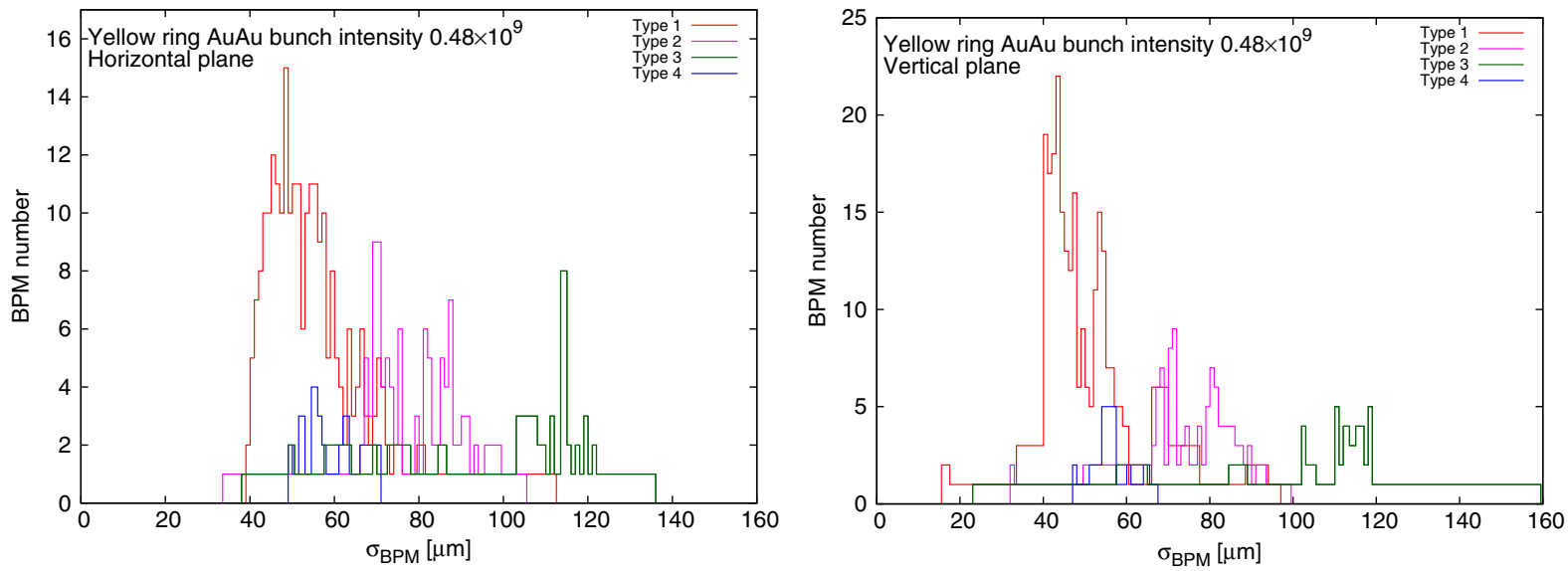

FIG. 5. Histograms of the estimated horizontal (left) and vertical (right) BPM noise corresponding to the case of Au-Au operation with a bunch intensity of $0.48 \times 10^{9}$ in Fig. 4 .

where $d$ is the beam pipe aperture and $V_{R}$ and $V_{L}$ are the voltage of the right and left BPM plates, respectively. Assuming the noises from the two BPM plates are independent, the thermal noise in beam position $x$ is estimated from Eq. (3) as

TABLE I. RHIC BPM mechanical details.

Type Planes Locations Stripline length [mm] Aperture [mm]

\begin{tabular}{lcccr}
\hline 1 & Single & IR and arc & 243.332 & 69.088 \\
2 & Dual & IR & 243.332 & 69.088 \\
3 & Dual & IR & 242.824 & 112.725 \\
4 & Dual & IR & 242.824 & 112.725 \\
\hline \hline
\end{tabular}

$$
\sigma_{x}=\frac{d}{4} \frac{\sqrt{2} \sigma_{v}}{V_{\Sigma}}=\frac{d}{\sqrt{2}} \frac{\sqrt{k_{\mathrm{B}} T R \Delta f}}{V_{\Sigma}},
$$

where $V_{\Sigma}=V_{R}+V_{L}$ is the sum signal which depends on the beam charge, bunch length, transfer impedance, and bandwidth. The model indicates that the noise level is proportional to the BPM aperture and is inversely proportional to the beam charge. To test this model, we normalize the BPM noise level of Fig. 4 to a factor of $d / C$, where $d$ is the BPM aperture and $C$ the beam charge in units of the charges in $1.0 \times 10^{9} \mathrm{Au}^{79+}$ particles. The normalized BPM noise distributions are shown in Fig. 6. All normalized BPM noise distributions 


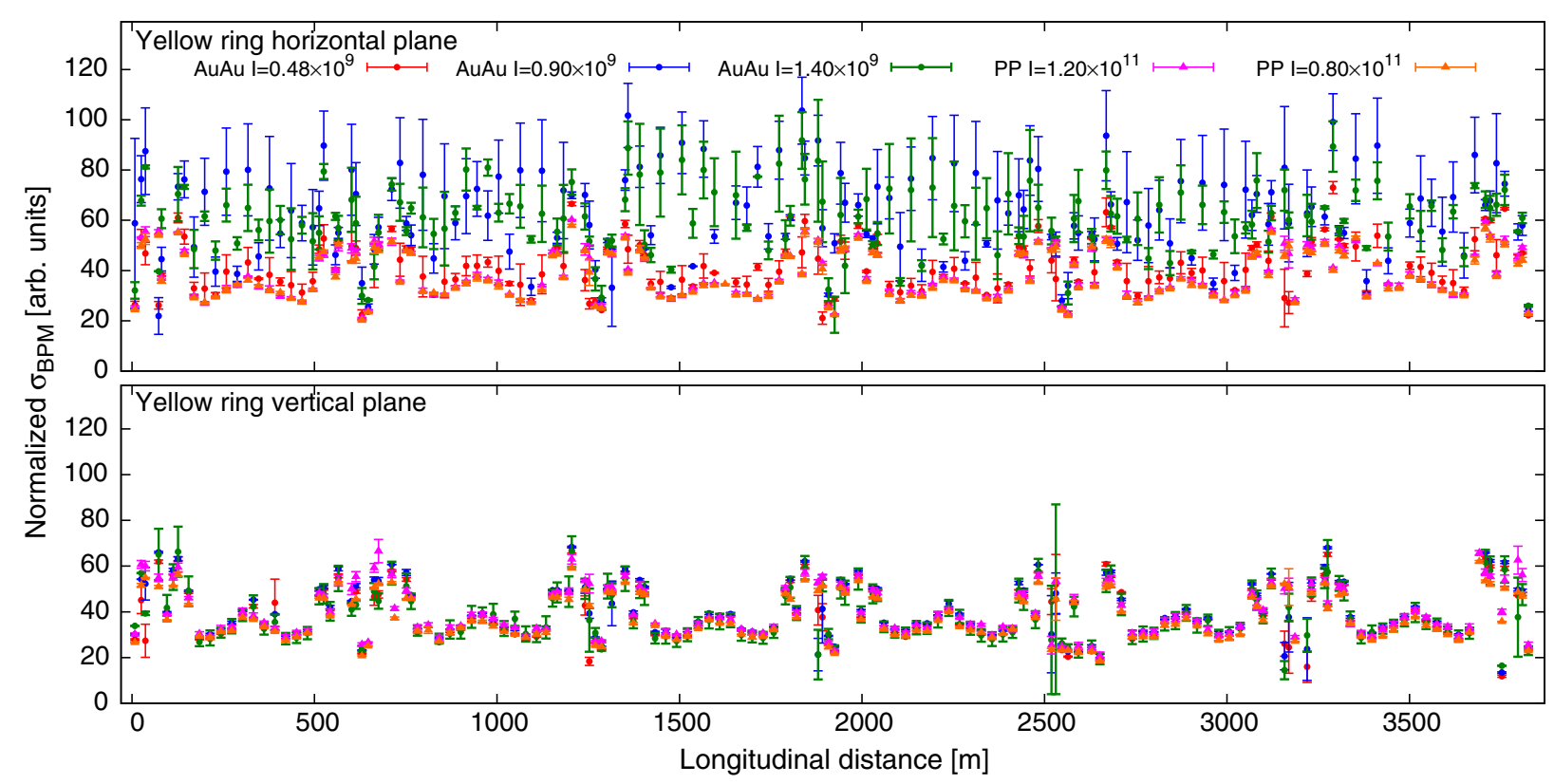

FIG. 6. Estimated noise in Fig. 4 normalized to the ratio of BPM aperture to beam charge (at $1 \times 10^{9}$ gold ion or $0.79 \times 10^{11}$ protons). Error bars reflect statistical standard deviations of estimated noise over three sets of experimental data.
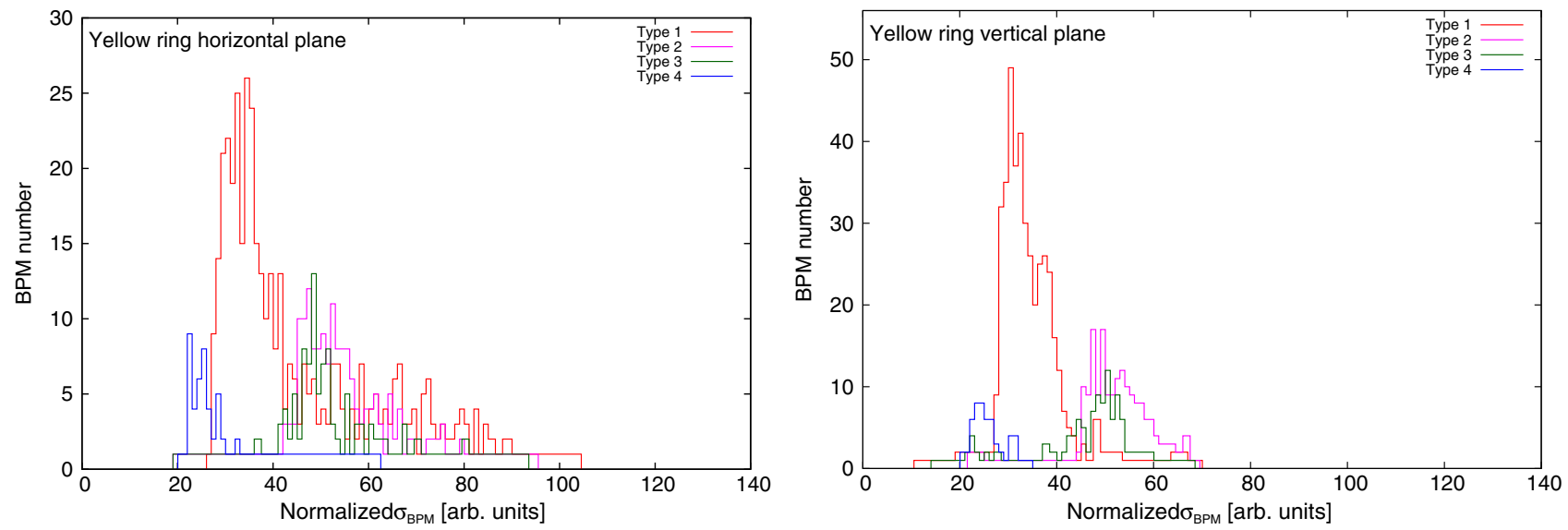

FIG. 7. Histograms of normalized noise in the horizontal (left) and vertical (right) BPMs for all cases in Fig. 6.

seem to collapse into nearly identical noise levels, except for the two cases of horizontal normalized noise of $\mathrm{AuAu}$ $0.90 \times 10^{9}$ and $1.40 \times 10^{9}$. Note that the error bars for these two cases are much larger than the others, which indicates a larger variation in the measurement data. This discrepancy may come from some unknown random noise when the data were taken.

Figure 7 shows histograms of the normalized BPM noise of all cases in Fig. 6. The normalized noise distributions of type 2 and 3 BPMs have a large overlapped portion. Note that apertures of type 1 and 2 BPMs are the same, but the average normalized noise of a type 2 BPM is larger than that of a type 1 BPM. This may be due to the fact that type 2 BPMs are dual plane, while type $1 \mathrm{BPMs}$ are single plane. Cross talk between two plates of a type 2 BPM can produce larger noise. Although type 3 and 4 BPMs have the same aperture, the average normalized noise of type 4 BPMs is about half of that of the type 3 BPMs, but within the spread of the type 3 BPMs. Notice that type 4 BPMs are located next to the beam merging dipoles and common to both the blue and yellow rings. The nonoverlapping part of the four types of distribution may arise from variations of the sum signal or the noise in each BPM. The variation of normalized noise for BPMs of the same type is possibly due to variation of the BPM parameters in Eq. (4).

\section{CONCLUSION}

An ICA algorithm is applied for absolute BPM noise level estimation from TBT BPM data. The capability of the ICA 
algorithm for noise estimation is validated by numerical simulations. Then the ICA algorithm was applied to experimental TBT BPM data taken during the RHIC 2013 polarized proton and $2014 \mathrm{Au}^{79+}$ operations for BPM noise estimation. The results show four types of characteristic noise level distributions, which is consistent with the current configuration of four types of BPMs. A thermal noise model is adopted to analyze the estimated noise distribution. A scaling law predicted by the thermal noise model is validated by experimentally estimated BPM noise. Further investigations are needed to improve the thermal noise model and find out the reason for unknown horizontal BPM noise for the AuAu $0.90 \times 10^{9}$ and $1.40 \times 10^{9}$ cases.

The BPM noise measurement can be carried out parasitically. With parasitic measurements, any unexpected ICA signal can be used as a warning sign or a characteristic for neuronetwork system to pinpoint possible problems in an accelerator. This BPM noise analysis is a powerful extension of the ICA method in beam diagnosis. In addition, this analysis based on 1024 TBT BPM data is immune to low frequency orbit vibrations, such as the $10 \mathrm{~Hz}$ vibration at RHIC [14].

\section{ACKNOWLEDGMENTS}

This work is supported in part by grants from the U.S. Department of Energy under Contract No. DE-FG0212ER41800 and the National Science Foundation NSF PHY-1205431. We thank Dr. H. Huang for his helpful discussions on the digitized sum signals at RHIC.
[1] A. Hyvarinen, J. Karhunen, and E. Oja, Independent Component Analysis (John Wiley \& Sons, New York, 2001).

[2] X. Pang and S. Y. Lee, J. Appl. Phys. 106, 074902 (2009).

[3] F. Wang and S. Y. Lee, Phys. Rev. ST Accel. Beams 11, 050701 (2008).

[4] X. Huang, S. Y. Lee, E. Prebys, and R. Tomlin, Phys. Rev. ST Accel. Beams 8, 064001 (2005).

[5] J. S. Kolski, R. J. Macek, R. C. McCrady, and X. Pang, Phys. Rev. ST Accel. Beams 15, 112802 (2012).

[6] X. Shen, S. Y. Lee, M. Bai, S. White, G. RobertDemolaize, Y. Luo, A. Marusic, and R. Tomás, Phys. Rev. ST Accel. Beams 16, 111001 (2013).

[7] C.-x. Wang, V. Sajaev, and C.-Y. Yao, Phys. Rev. ST Accel. Beams 6, 104001 (2003).

[8] R. Calaga and R. Tomás, Phys. Rev. ST Accel. Beams 7, 042801 (2004).

[9] S. De Santis, M. Chin, D. Filippetto, W. Norum, Z. Paret, and G. Portman, in Proceedings of the 2nd International Beam Instrumentation Conference, IBIC-2013, Oxford, $U K$, edited by I. Martin and G. Rehm (Oxford, UK, 2013), pp. 108-111.

[10] M. Bai, S. Y. Lee, J. W. Glenn, H. Huang, L. Ratner, T. Roser, M. J. Syphers, and W. van Asselt, Phys. Rev. E 56, 6002 (1997).

[11] T. Satogata et al., in Proceedings of the 21st Particle Accelerator Conference, Knoxville, TN, 2005 (IEEE, Piscataway, NJ, 2005), pp. 2021-2023.

[12] J. B. Johnson, Phys. Rev. 32, 97 (1928).

[13] R. E. Shafer, AIP Conf. Ser. 212, 26 (1990).

[14] R. Michnoff et al., in Proceedings of the 24th Particle Accelerator Conference, PAC-2011, New York, 2011 (IEEE, New York, 2011). 